\title{
A Study on Prevalence of Escherichia coli 0157 with a Verified Method in Foods
}

\author{
Semiha Yalçın ${ }^{1 *}$, Ayla Ünver Alçay ${ }^{2}$, Gözde Yüzbaşığlư ${ }^{1}$, Burcu Çakmak ${ }^{3}$, Aysun Sağlam ${ }^{4}$ \\ ${ }^{1}$ Marmara Food Analysis Laboratory, Istanbul Aydin University, 34295 Istanbul, Turkey \\ ${ }^{2}$ Department of Food Technology, Istanbul Aydin University, 34295 Istanbul, Turkey \\ ${ }^{3}$ Agrolab Food Analysis Laboratory, 34394 Istanbul, Turkey \\ ${ }^{4}$ Department of Food Quality Control and Analysis, Istanbul Aydin University, 34295 Istanbul, Turkey
}

A R T I C LE IN F O

\section{Research Article}

Received 04 October 2016

Accepted 07 March 2017

Keywords:

E. coli $\mathrm{O} 157$

Prevalence

Food pathogen

Verification

Esherichia coli \begin{abstract}
A B S T R A C T
The purpose of this study were to identify the presence of E.coli O157 and to determine its prevalence in foods which were collected from various restaurants, shops and markets in Istanbul. Also, validation of detection method of E. coli $O 157$ in all food stuffs was carried out according to applicability, repeatability, and minimum detection limit (LOD) and false positive and negative analysis based on TS EN ISO 16654 standard method. The results showed that the prevalence of E. coli $\mathrm{O} 157$ in food was $2 \%$, and its prevalence increased in April and May.
\end{abstract}

*Corresponding Author:

E-mail: semihayalcin@aydin.edu.tr

\section{Introduction}

Escherichia coli is a bacterium that normally presents in the intestines of humans and warm-blooded animals. Most of its strains are harmless and actually are necessary for human intestinal flora. However, pathogenic strains of $E$. coli cause serious infections in humans and animals. These pathogenic variants of $E$. coli can be transmitted by mainly contaminated meat and meat products, raw milk, fruits, vegetables, water and environmental resources (Anonymous, 2011; Anonymous, 2014).

Escherichia coli belongs to Enterobacteriaceae family. There are six of E. coli pathotypes cause immense outbreaks in the world, such as Enteropathogenic E. coli (EPEC), Shigatoxin or Verotoxin producing E. coli (STEC/VTEC), Enteroinvasiv E. coli (EIEC), Enteroaggregative E. coli (EAEC), Diffusely Adherent $E$. coli (DAEC), Enterotoxigenic E. coli (ETEC) and a new pathotypes identified as adherent-invasive E. coli (AIEC). STEC/VTEC is also known as Enterohaemorrhagic $E$. coli (EHEC) (Anonymous, 2014; Croxen et al., 2013).

Verotoxin producing E. coli (VTEC) have been identified for the first time in 1970's by Konowalchuk and co-workers in Canada (Konowalchuk et al., 1977). The lethal toxin for HeLa cells in the polypeptide structure identified by O'Brien and LaVeck in 1983 was obtained from strains of verotoxin producing E. coli. Toxin is called shiga-like toxin because of similarity in its structural and antigenic characteristics with Shigella dysenteriae type 1 toxsin (O'Brien et al., 1983).

Verotoxin producing E. coli (VTEC) is an important pathogen that present in foods and water resources, which causes epidemic diseases such as diarrhoea, haemorrhagic colitis and haemolytic uremic syndrome. There are over $380 \mathrm{OH}$ serotypes of E. coli (VTEC). Nevertheless, serotypes which cause serious infections in humans are limited. O26, O91, O103, O111, O113, O121, O145 and O157 serotypes are important infection treats. Among those, the most common infection causing serotype is $E$. coli O157:H7 serotype. Therefore, VTEC serotypes are classified as E. coli $O 157$ and non-E. coli O157. E. coli $O 157$ is called Enterohaemorrhagic E. coli (EHEC) (Karmali et al., 2010).

Symptoms in the EHEC infection are severe abdominal pain and bloody diarrhea. Usually not exceeding $38.5^{\circ} \mathrm{C}$ fever and vomiting may occur. The haemolytic uremic syndrome (HUS) can develop at more than $10 \%$ of patients, and can result in death of $3-5 \%$ of these cases with the HUS table. It can be seen acute renal failure in cases of children. The symptoms of the disease 
arise in 3-4 days after exposure, after average 7 days. The incubation period may vary between 1-10 days. Various antibiotics are used for the treatment (Anonymous, 2011; Anonymous, 2014).

E. coli $\mathrm{O} 157$ can be detected by using PCR (Polymerase Chain Reaction) and immunospesific methods; classical serological and culture methods are also commonly used in laboratory (Karmali et al., 2010).

In this study, prevalence of E. coli O157 in foods in Istanbul was performed by classical culture method which was verified according to TS EN ISO 16654. Repeatability, the limit of detection (LOD), false positive rate, false negative rate, specificity, sensitivity studies were evaluated for method verification (Eurachem Guide AML, Health Protection Agency Guidance Note (NHS), QSOP 22).

\section{Materials and Methods}

\section{Method Verification Studies}

Stock preparation and sampling: Samples were selected according to ISO 19036 as infant formula, mayonnaise, dog food and mold cheese. E. coli 0157 ATCC 700728 (CECT) strain was used as positive control, and Enterococcus faecalis ATCC 19433 (Liofilchem) was used as negative control according to ISO 11133 directive. Stock solution was prepared for artificial contamination of selected foods with $E$. coli O157 ATCC 700728 strain. Mac Farland (Biomerieux) standard was used for determining microorganism number and then serial dilutions were prepared for obtaining 2 $\mathrm{cfu} / \mathrm{ml}$ and $100 \mathrm{cfu} / \mathrm{ml}$ for revealing limit of detection and detection of high level microorganism. Studies were carried out by two analysts for ensuring reproducibility of the method.

Method: Samples were homogenized with sterilized blender. Homogenized sample was weighed $25 \mathrm{~g}$ in stomacher filter bag. Samples were artificially contaminated with E. coli O157 ATCC 700728 and Enterococcus faecalis ATCC 19433 with using stock solution after that $225 \mathrm{ml}$ tryptone soya broth (added novobiocin) (LabM) was added to sample for preenrichment at $41.5^{\circ} \mathrm{C}$ for 6 hours. For selection of pure form of E. coli $\mathrm{O} 157$ from the pre-enrichment medium, immunomagnetic separation process was performed according to the Captivate ${ }^{\mathrm{TM}} \mathrm{O} 157$ (Lab M-CAP001) protocol. After the separation process, $50 \mu \mathrm{l}$ of sample was inoculated in Sorbitol Mac Conkey Agar (CTSMAC) (LabM) that prepared with adding Cefixime and potassium tellurite. Also, it was inoculated in TC-SMAC agar. Colonies were incubated for $18-24$ hours at $37^{\circ} \mathrm{C}$. Typical colonies of $E$. coli $O 157$ were observed as transparent, light yellowish-brown and about $1 \mathrm{~mm}$ in diameter in TC-SMAC agar. 5 typical 0157 colonies were selected and inoculated to Nutrient Agar then incubated for $18-24$ hours at $37^{\circ} \mathrm{C}$. Pure cultures were obtained from nutrient agar to be used for biochemical identification such as oxidase, catalase tests and Gram reaction. Gram-negative, bacilli form, oxidase negative, catalase positive colonies were selected for biochemical identification. Biochemical characteristics of the bacteria were determined by GNA A-B biochemical kit (Microgen) and E. coli was identified. For identification of E. coli O157, latex agglutination test (Microgen) was used according to the manufacturer instruction.

Repeatability, false negative rate, false positive rate and lod study: Analyses were carried out by two analysts for ensuring repeatability in same day at short intervals. 10 different sampling were performed for each of 4 different product groups (infant formula, moldy cheese, $\operatorname{dog}$ food and mayonnaise) which were artificially contaminated with E. coli O157 at LOD level (2 cfu/25 g) and also at high level (100 cfu/25 g). False negative rate study was performed at these two levels as high and low. Artificially contaminated with Enterococcus faecalis samples were analysed for false positive rate study.

\section{Prevalence of E. coli O157 Study}

Isolation and identification of E. Coli O157 strains: Total of 200 samples were collected randomly in April 2015 and in January-May 2016 from 63 different food companies in Istanbul as shown in Table 1.

All analyses were carried out according to international standards TS EN ISO 16654 classical method.

\section{Results}

In this study, determination and identification of Escherichia coli O157 was carried out according to verified TS EN ISO 16654 method in foods. Our test results showed that false negative and false positive analysis results were calculated as $\% 0$, relative sensitivity, relative accuracy and relative specificity analysis results were calculated as \%100. Verification of TS EN ISO 16654 method study was evaluated as acceptable when interpreted in compliance with the Kappa (K) method (Table 2) (NMKL Procedure No. 20). Besides, for accuracy study, we participated to international proficiency test round for E. coli O157 screening analysis. Proficiency test results were appropriate.

In this research, also E. coli $O 157$ was screened in various food samples apart from the verification study. Nine samples were collected from different markets in 2015 April and two of them were positive for $E$. coli O157. E. coli $O 157$ was detected in 2 out of 10 samples picked from different food production areas in May 2016. In April 2015, raw chicken obtained from chicken shredding facility and ground beef samples obtained from a butcher were positive for E. coli O157. Among the samples collected in May 2016, raw beef mince and garnish were positive for E. coli O157. The prevalence of this pathogen in 200 food samples was determined as $2 \%$.

\section{Discussion}

Food control laboratories have been authorized for detection of such pathogens and other elements in foodstuffs that risk public health by Ministry of Food, Agriculture and Livestock. This study was carried out in 
accordance with international standards analysis for fulfilling the obligation for authorization process. Only accredited laboratories are authorized. In the accreditation process, after verification of all laboratory analyses, verification studies are attested by TURKAK (Turkish
Accreditation Agency) according to international validity of the scope of TS EN ISO/IEC 17025 (Eurachem Guide AML, Health Protection Agency Guidance Note (NHS), QSOP 22, Turkish Food Codex Microbiological Criteria Regulation, 2011).

Table1 Collected samples

\begin{tabular}{|c|c|c|c|}
\hline Sample & NS & Sample & NS \\
\hline Chicken meat (raw) & 5 & Mayonnaise & 1 \\
\hline Mediterranean salad & 2 & Meatballs with vegetable (raw) & 1 \\
\hline Chicken meatballs (raw) & 1 & Meat $* * *$ doner with souce & 1 \\
\hline Chicken schnitzel (raw) & 1 & Garnish & 4 \\
\hline Chicken burger (raw) & 1 & Minced veal (raw) & 36 \\
\hline Chicken nugget (raw) & 1 & **Lahmacun minced meat (raw) & 2 \\
\hline Sheep lard (raw) & 1 & Minced lamb (raw) & 4 \\
\hline Veal carcass (raw) & 7 & Part of lamb meat (raw) & 5 \\
\hline Veal part of meat (raw) & 17 & Lamb carcass (raw) & 3 \\
\hline Cubed veal (raw) & 5 & Veal liver (raw) & 2 \\
\hline Cooked chicken $* * *$ doner & 4 & Sauce & 1 \\
\hline Minced meat (raw) & 6 & Cake & 1 \\
\hline Veal rib meat (raw) & 1 & Veal meat with souce (raw) & 1 \\
\hline Cooked meat $* * *$ doner & 1 & Frozen red pepper & 1 \\
\hline Meatballs (raw) & 44 & Frozen diced tomatoes & 1 \\
\hline *Inegol meatballs (raw) & 4 & Veal tenderloin (raw) & 2 \\
\hline Grilled meatballs (raw) & 9 & Veal arm (raw) & 1 \\
\hline Hamburger meatballs (raw) & 15 & Lamb's shank (raw) & 1 \\
\hline Shrimp (raw) & 1 & *Adana meatballs (raw) & 1 \\
\hline American salad & 1 & Fish (raw) & 1 \\
\hline Cucumber & 1 & Sausage & 2 \\
\hline Total & & & 200 \\
\hline
\end{tabular}

NS: Number of sample, *A kind of Turkish traditional meatball. **A kind of Turkish fast food with very thin dough, like pizza. ***A kind of Turkish fast food.

Table 2 Results for kappa evaluation

\begin{tabular}{|c|c|c|c|}
\hline Verification Samples & Positive Result & Negative Result & Total \\
\hline E. coli $O 157$ inoculated samples (positive samples) & $160(a)$ & $0(\mathrm{~b})$ & $160(a+b)$ \\
\hline Samples that not include $E$. coli $O 157$ (negative samples) & $0(\mathrm{c})$ & $80(d)$ & $80(c+d)$ \\
\hline Total & $160(a+c)$ & $80(b+d)$ & $240(\mathrm{n}=\mathrm{a}+\mathrm{b}+\mathrm{c}+\mathrm{d})$ \\
\hline
\end{tabular}

$a$ : E. coli $\mathrm{O} 157$ inoculated samples and positive identified samples, b:İnoculated samples but identified as negative, c: Not inoculated but identified as positive samples, d: Only Enterococcus faecal is inoculated but identified as negative samples, n: Total number of samples, Relative Sensitivity: $\mathrm{a} /(\mathrm{a}+\mathrm{b}) \times 100=160 / 160 \times 100=\% 100$ S Relative Accuracy: $\mathrm{a}+\mathrm{d} / \mathrm{n} \times 100=240 / 240 \times 100=\% 100$, Relative Specificity: $\mathrm{d} / \mathrm{c}+\mathrm{dx} 100=80 / 80 \times 100=$ $\% 100$, False negative rate: $\mathrm{b} / \mathrm{b}+\mathrm{a}=0 / 160=\% 0$, False positive rate: $\mathrm{c} / \mathrm{c}+\mathrm{dx} 100=0 / 80 \times 100=\% 0$

E. coli $O 157$ is a serious threat to public health in Europe and America that led to the outbreak. In our country, the Turkish Food Codex, Microbiological Criteria for food safety was published by the Ministry of Food, Agriculture and Livestock. According to this regulation; particularly meat and meat products, fermented meat products, fruits and vegetables, including processed products and ready to consumption food, $E$. coli $O 157$ certainly must not be present (Turkish Food Codex Microbiological Criteria Regulation, 2011).

The presence of $E$. coli in Turkey was demonstrated in many studies before. E. coli $\mathrm{O} 157$ was investigated in 150 samples of calf minced and lamb minced meat collected from retail markets and butcheries and E.coli O157 was found in $6.66 \%$ in total of 300 samples in Van (Alişarlı and Akman, 2004). And also in Afyonkarahisar E. coli O157:H7 presence was shown in raw milk and cheese samples (Akkaya et al., 2007). Although registrations and information about prevalence and incidence of STEC strains are limited in Turkey (Bostan et al., 2005). In our study, 186 meat products and 14 salads including sauce samples were investigated for E.coli 0157 presence and detected in 3 meat products including 1 cooked meat sample and in 1 garniture sample.

In Turkey, E. coli O157:H7 were not isolated from 100 pieces hamburgers (Sarımehmetoğlu et al., 1998). 127 samples of frozen hamburger and meatballs were screened for E. coli O157:H7, and E. coli O157: H7 was isolated from 3 samples (O’Brien and LaVeck, 1983). On the other hand, in our study, E. coli O157 was detected in raw chicken and raw mince samples and also cooked chicken, ready-to-eat garniture sample.

In Konya, sausage, salami, hamburger meat, Inegol meat ball, pastrami, minced meat and brisket and haunch of poultry were collected from different meat sellers, and analyzed for presence of E. coli $O 157$ and coliform 
bacteria. E. coli $O 157(11.1 \%)$ was determined, in two of them E.coli $0157: H 7(8.1 \%)$ and in one refrigerated hamburger meat ball sample E. coli $O 157$ and E.coli O157:H7 (4.34\%) were determined (Balpetek and Gürbüz, 2010).

In another study in Turkey in Aydin, a study was carried out for detecting Escherichia coli O157:H7 serotype in minced beef and uncooked beef burgers collected from butcher shops and supermarkets. E. coli $O 157$ serotypes were isolated from 6 minced beef and 6 uncooked hamburger meatballs by conventional culture techniques (Sezgin, 2013).

In Samsun, a total of 200 samples collected from cattle carcasses and the rectal contents of 100 slaughtered cattle from two commercial abattoirs were analyzed for Escherichia coli $O 157$ and E. coli O157:H7 using immunomagnetic separation technique and multiplex PCR methods. E. coli $O 157$ and E. coli O157:H7 were detected in 52 out of 200 samples $(26 \%)$ tested. Of the positive samples, 49 were $E$. coli $O 157$ and three were $E$. coli O157:H7. The stx 1 and stx 2 genes were both detected in 35 E. coli $O 157$ isolates and one E. coli O157:H7 isolate, but the stx 2 gene was only detected in two $E$. coli O157 isolates (İnat and Siriken, 2013).

Microbiological properties of 25 chicken breast and 25 thigh sold in Tokat were tested. According to the results; E. coli O157:H7 were isolated form 6 (24\%) chicken breast and $6(24 \%)$ chicken thigh samples (Y1ldirım et al., 2015).

30 samples, each of ready-to-cook meatballs and white cheese as well as 96 samples of various ready-to-eat foods (paste with hot pepper, American salad, cold white beans vinaigrette (barbunya pilaki), kisir, fried eggplant, fava, haydari, humus, sarma, mushroom salad, pasa mezesi, vegetable salad with yogurt, tarator) were collected from different outlets in Istanbul, and were investigated for the presence of verotoxins (consequently verotoxigenic $E$. coli) with immunoassay. Verotoxins were not detected in any of the tested samples (Bostan et al., 2005).

Examples of studies in the Middle East countries; during a period from March 2010 to March 2011, prevalence of E. coli $0157: H 7 / N M$ in raw meat samples (beef, camel, sheep, goat, and water buffalo meat) was studied in Iran, and E. coli $O 157$ was found in beef samples $(8.2 \%)$, water buffalo $(5.3 \%)$, sheep $(4.8 \%)$, camel $(2.0 \%)$, and goat meat $(1.7 \%)$. One out of 14 samples was determined to be serotype $0157: H 7$ and the others were determined as serotype O157:NM. Also stxl, stx2, eaeA and ehlyA genes were detected in 4 strains. It is demonstrated that the prevalence of $E$. coli $O 157$ occurred at the highest level in the summer $(9.3 \%)$. It is emerged that in Iran, the beef and water buffalo meat are a significant source for $E$. coli O157:H7/NM infection (Rahimi et al., 2012).

The results of our study showed that prevalence of $E$. coli 0157 increased in April and May. In January, February and March, the samples taken from the 46 companies were found as negative for $E$. coli $O 157$.
These companies such as caterings, restaurants were periodically checked for food production.

It was determined that the requirements of HACCP rules, hygiene criteria of preservation and storage conditions for foods must be fulfilled for eliminating $E$. coli $O 157$ and other pathogens with regular analysis carried out by authorized and accredited laboratories.

\section{References}

Alişarlı M, Akman HN. 2004. Examining the direction of Escherichia coli 0157 in ground beef sold in retail. Van Vet. J. 5(1): 65-69.

Akkaya L, Alisarli M, Kara R, Telli R. 2007. The Prevalance of $E$. coli O157:H7 in Raw Milks and Cheeses Sold in Afyonkarahisar Province, Van Vet J. 18(1): 1-5.

Anonymous. 2011. Enterohaemorrhagic Escherichia coli (EHEC): a conceptual framework. World Health Organization. Accessed: 24.03.2015 Avaliable: http://www.who.int/mediacentre/ factsheets/fs 125/en/

Anonymous. 2014. E. coli (Escherichia coli): a conceptual framework. Center for Disease Control and Prevention. Accessed: 24.03.2015 Available: http://www.cdc.gov/ecoli/ general/

Balpetek D, Gürbüz U. 2010. Investigation of the presence of E. coli 0157: $H 7$ in some meat products. Eurasian J. Vet. Sci. 26(1): 25-31

Bostan K, Çetin O, Ergün O. 2005. The presence of verotoxinogenic E. coli in some foods. Vet. Glasnik. 59 (3-4): 427-436.

Bozcal E, Yiğittürk G, Uzel A, Aydemir SŞ. 2016. Investigation of enteropathogenic Escherichia coli and Shiga toxin-producing Escherichia coli associated with hemolytic uremic syndrome in İzmir Province, Turkey. Türk. J. Med. Sci. 46(3): 733-41

Cebiroğlu H, Nazlı B. 1999. Research studies, being on enterohaemorrhagic Escherichia coli 0157.H7 in frozen hamburgers, meatballs and other meatballs type. İstanbul Univ. Vet. Fak. Derg. 25(1): 107-121.

Croxen MA, Law RJ, Scholz R, Keeney KM, Wlodarska M, Finlay BB. 2013. Recent Advances in Understanding Enteric Pathogenic Escherichia coli. Clin. Microbiol. Rev. 26(4): 822880 .

Eurachem Guide AML. 2013. Accreditation for Microbiological Laboratory.

Health Protection Agency Guidance Note (NHS), QSOP 22. 2005. Minimum performance testing requirements for food and dairy standard methods.

Hessain AM, Al-Arfaj AA, Zakri AM, El-Jakee JK, Al-Zogibi OG, Hemeg HA, İbrahim IM. 2015. Molecular characterization of Escherichia coli O157:H7 recovered from meat and meat products relevant to human health in Riyadh, Saudi Arabia. Saudi J. Biol. Sci. 22(6): 725-9

İnat G, Siriken B. 2010. Detection of Escherichia coli O157 and Escherichia coli O157:H7 by the immunomagnetic separation technique and stx1 and stx2 genes by multiplex PCR in slaughtered cattle in Samsun Province, Turkey. J. Vet. Sci. 11(4): 321-6.

ISO 11133. 2014. Microbiology of food, animal feed and water Preparation, production, storage and performance testing of culture media.

Karmali MA, Gannon V, Sargeant JM. 2010. Verocytotoxinproducing Escherichia coli (VTEC). Vet. Microbiol. 40(3-4): 360-370.

Konowalchuk J, Speirs JI, Stavric S. 1997. Vero response to a cytotoxin of Escherichia coli. Infect. Immun. 18: 775-77.

NMKL Procedure No. 20. 2007. Evaluation of results from Qualitative methods.

O'Brien AD, LaVeck GD. 1983. Purification and characterization of a Shigella-dysenteriae 1-like toxin produced by Escherichia coli. Infect. Immun. 40: 675-683. 
Rahimi E, Kazemeini HR, Salajegheh M. 2012. Escherichia coli O157:H7/NM prevalence in raw beef, camel, sheep, goat, and water buffalo meat in Fars and Khuzestan provinces, İran. Vet. Res. Forum. 3(1): 15-7.

Sarımehmetoğlu B. Kuplulu O, Kaymaz S. 1997. Escherichia coli O157:H7 isolation from hamburgers and Inegol Meatballs. Ankara Univ.Vet. Fak. Derg. 45: 221-227.

Sezgin E. 2013. Investigating of the presence of Escherichia coli O157:H7 in minced beefand hamburger meatballs which consumed in Aydin region. M. Sc. Thesis. Adnan Menderes University. Aydın.
TS EN ISO 16654. 2003. Microbiology of food and animal fodderhorizontal method for the detection of E. coli O157.

TS/ISO 19036. 2006. 2009(E) Amd-1. Microbiology of food and animal feding stuffs, Guidelines for the estimation of measurement uncertainty for quantitative determinations.

Turkish Food Codex Microbiological Criteria Regulation. 29.12.2011. Ministry of Food, Agriculture and Livestock.

Yıldırım Z, Ceylan Ş, Öncül N. 2015. Microbiological Quality of Chicken Breast and Thigh Samples Sold in Tokat, Turkey. Akademik Gıda. 13(4): 304-316. 